TITLE:

\title{
Hydrodynamic radius of circular DNA is larger than that of linear DNA
}

$\operatorname{AUTHOR}(\mathrm{S}):$

Araki, Sumiko; Nakai, Tonau; Hizume, Kohji;

Takeyasu, Kunio; Yoshikawa, Kenichi

CITATION:

Araki, Sumiko ...[et al]. Hydrodynamic radius of circular DNA is larger than that of linear DNA. Chemical Physics Letters 2006, 418(1-3): 255259

ISSUE DATE:

2006-01

URL:

http://hdl.handle.net/2433/49162

RIGHT:

Copyright (C) 2005 Elsevier B.V. All rights reserved.; この論文は出版社版 でありません。引用の際には出版社版をご確認ご利用ください。; This is not the published version. Please cite only the published version. 


\title{
Hydrodynamic radius of circular DNA is larger than that of linear DNA
}

\author{
Sumiko Araki ${ }^{a}$, Tonau Nakai ${ }^{\mathrm{a}}$, Kohji Hizume ${ }^{\mathrm{b}}$, \\ Kunio Takeyasu ${ }^{b}$, Kenichi Yoshikawa ${ }^{a, *}$ \\ ${ }^{a}$ Department of Physics, Graduate School of Science, Kyoto University, Kyoto \\ 606-8502, Japan \\ ${ }^{\mathrm{b}}$ Laboratory of Plasma Membrane and Nuclear Signaling, Graduate School of \\ Biostudies, Kyoto University, Kyoto 606-8502, Japan
}

\begin{abstract}
It is generally accepted that linear polymers are larger, such as with regard to the end-to-end distance and gyration radius, than the corresponding circular polymers. We measured the Brownian motion of individual linear and circular 106 kilo basepair (kbp) DNA molecules using fluorescence microscopy (FM). Unexpectedly, the hydrodynamic radius of linear DNA was markedly smaller than that of circular DNA.
\end{abstract}

Key words: circular chain, hydrodynamic radius, giant DNA

\section{Introduction}

The conformation of single circular chains is a fundamental problem in polymer physics [1]. Nevertheless, there have been only few experiments on circular chains because it is difficult to synthesize a closed loop. The observation of viral DNA, which exists in nature as a circular chain, has stimulated several studies on ring polymers [2-4].

In an ideal linear chain, the mean-square end-to-end distance $L^{2}$ is given by

$$
<L^{2}>=b^{2} N
$$

\footnotetext{
* Corresponding author. Fax: +81-75-753-3779.

e-mail: yoshikaw@scphys.kyoto-u.ac.jp (K. Yoshikawa).
} 
where $b$ is the Kuhn segment and $N$ is the number of segments. The gyration radius $R_{\mathrm{g}}$ is

$$
<R_{\mathrm{g}}^{2}>=<L^{2}>/ 6=b^{2} N / 6
$$

For an ideal circular chain, $L=0$ by definition, and the gyration radius is deduced as [1]

$$
<R_{\mathrm{g}}^{2}>_{\text {circular }}=b^{2} N / 12=<R_{\mathrm{g}}^{2}>_{\text {linear }} / 2 .
$$

This indicates that the gyration radius of an ideal circular chain is smaller than that of a linear chain of the same contour length by a factor of $\sqrt{2}$. Jang et al. extended this relationship to polymers with an excluded volume as follows citePEring:

$$
<R_{\mathrm{g}}^{2}>_{\text {circular }}=<R_{\mathrm{g}}^{2}>_{\text {linear }}\left(1+\frac{\zeta}{3+2 \zeta}\right) / 2
$$

where $\zeta$, the excluded volume parameter, is defined as $\zeta=2 \nu-1$ and $\nu$ is the Flory exponent. On the other hand, the hydrodynamic radius $R_{\mathbf{H}}$ in an ideal linear chain, defined as the radius of a hydrodynamically equivalent sphere, is expected to be smaller than $R_{\mathbf{g}}$ following the Zimm model [6];

$$
R_{\mathrm{g}} \approx 1.24 R_{\mathbf{H}}
$$

A similar relationship between $R_{\mathbf{g}}$ and $R_{\mathbf{H}}$ has been considered to be valid in general for real chains with an excluded volume $[7,8]$. In a theoretical paper, it has also been reported that $R_{\mathbf{H}}$ is proportional to $R_{\mathrm{g}}$ in circular chains and that the $R_{\mathbf{H}}$ of a circular chain is smaller than that of a linear chain [9]. Thus, it is generally thought that the $R_{\mathbf{H}}$ of a linear chain should exceed that of a circular chain with the same contour length, for both ideal and real polymers. To the best of our knowledge, however, this theoretical expectation has not yet been verified experimentally, partly due to the difficulty of synthesizing circular chains and also due to the difficulty of observing the conformation of individual single closed chains in solution.

The conformation of linear polymer chains has been extensively studied by various experimental methods, such as X-ray or neutron scattering and dynamic light scattering, and theoretical studies have successfully described the observed chain behavior [10]. However, these methods are not particularly suitable for long polymers, such as genomic DNA with a contour length of more than $10 \mu \mathrm{m}$. Long flexible or semiflexible chains in dilute solution are not dense enough to be scattered, i.e., a DNA solution that is to be observed with a scattering method frequently exceeds the overlap threshold, and the extraction of this information from experimental data is a difficult problem that has various solutions, among which only one corresponds to physical reality. On the other hand, a powerful method for observing DNA in dilute solution and resolving these problems, is fluorescence microscopy $(\mathrm{FM})$, which makes it possible to observe Brownian motion and to obtain information on the structure 
of individual molecular chains in solution, although FM can only be applied to giant DNA molecules larger than several tens of kilo base pairs (kbp) [11]. In this study, we have used this method to measure the hydrodynamic radii of linear or circular DNA in dilute solution. In the present article, we report the conformational characteristics of linear and circular DNA molecules with a monodisperse size of $106 \mathrm{kbp}$. These DNA molecules were stained with fluorescence dye and observed by FM. The Brownian motion of these molecular chains was observed in real space and real time, due to the large contour length of $36 \mu \mathrm{m}$.

\section{Materials and methods}

The 106-kbp supercoiled plasmid DNA (sample A) was purified as reported [12]. The contour length (bp) of DNA we use was $106 \mathrm{kbp}$ throughout this study. The relaxed circular plasmid (sample B) was prepared by treating the supercoiled plasmid with type I topoisomerase (Promega). To prepare linearized DNA (sample C), the 106-kbp plasmid DNA was treated with the restriction enzyme, BstE II, which digests only one site. After the enzyme treatment, DNA was purified by phenol extraction and ethanol precipitation.

Field Inversion Gel Electrophoresis (FIGE) in $0.5 \times$ TBE buffer $(45 \mathrm{mmol} / \mathrm{L}$ tris(hydromethyl)aminomethane, $45 \mathrm{mmol} / \mathrm{L}$ boric acid, and $1 \mathrm{mmol} / \mathrm{L}$ EDTA, $\mathrm{pH}$ 8.3) was perfomed as described [13]. The gel chamber (CHEF Mapper, BioRad) with the agarose gel $(0.75 \%$, BioRad) was maintained at $283 \mathrm{~K}$. The electrophoresis $(3 \mathrm{~V} / \mathrm{cm})$ schedule was as follows : forward pulse time $30 \mathrm{~s}$, reverse pulse time $10 \mathrm{~s}$ and total pulse time $22 \mathrm{~h}$. After running, the gels were stained by immersion in a solution containing a fluorescent dye (SYBR Gold; Molecular Probes).

The 106-kbp DNA was dissolved at $0.2 \mu \mathrm{M}$ (base units) in a $25 \mathrm{mM}$ Tris- $\mathrm{HCl}$ buffer solution ( $\mathrm{pH} 7.5$ ) throughout this study. Each DNA was stained by a fluorescent dye (YOYO-1 $0.01 \mu \mathrm{M}$ ), and $8 \mu \mathrm{L}$ was adsorbed onto freshly cleaved mica, coated with $1 \mathrm{mM}$ spermidine and washed with Milli-Q-purified water for $4 \mathrm{~min}$. The mica surface was washed again with Milli-Q-purified water and dried in $\mathrm{N}_{2}$ gas. An NVB 100 (Olympus, Tokyo) operated in tapping mode was used. Images were displayed with flattening to remove the background curvature of the mica surface. The DNA conformation along the contour was digitized on the two-dimensional surface, $\boldsymbol{r}_{i}(i=1, N)$. Then, we calculated the radius of gyration $R_{\mathrm{g}}$ as follows:

$$
R_{\mathrm{g}}=\sum_{i=1}^{N}\left(\boldsymbol{r}_{i}-\boldsymbol{r}_{\mathrm{G}}\right)^{2} / N,
$$


where $\boldsymbol{r}_{\mathrm{G}}$ is the center of mass.

The 106-kbp DNA was visualized by fluorescence microscopy (FM) using a fluorescent dye, $0.1 \mu \mathrm{M}$ 4',6-diamidino-2-phenylindole (DAPI, Wako Chemical Industries). FM measurements were performed as follows. The experiments were illuminated with 365-nm UV light, and fluorescence images of DNA molecules were observed using a Carl Zeiss Axiovert 135 TV microscope equipped with a $100 \times$ oil-immersed objective lens. Images were recorded on a videotape at 30 frames per second through a high-sensitivity EB-CCD camera with an image-processing system (Hamamatsu Photonics). The video data were analyzed with personal computers. Due to the blurring effect [14], in the observation with a highly sensitive video system, the observed DNA images were assumed to be slightly larger $(\approx 0.3 \mu \mathrm{m})$ than the actual size of DNA. To evaluate the hydrodynamic radius of DNA in bulk solution, we measured the Brownian motion of individual DNA molecules using FM.

\section{Results and discussion}

\subsection{FIGE measurements}

FIGE measurement is suitable for separating Giant DNA with different topological forms [13]. Using this method, we investigated the form distribution in each solution. Figure 1 shows that sample $\mathrm{C}$ contained only the linear form, whereas samples A and B were unexpectedly mixtures of three forms. To distinguish the supercoiled form and relaxed form, we have estimated the percentages of each of the three forms in samples A and B. Based on an analysis of the fluorescence intensity, the percentages of supercoiled, relaxed and linear forms in sample A are 38, 30 and $32 \%$ and those in sample B are 30, 32 and $38 \%$, respectively.

\subsection{AFM measurements}

Typical AFM images of DNA in samples A, B and C, adsorbed on mica are shown in Fig. 2. Since the contour length of DNA is much larger than the persistence length $(\approx 0.05 \mu \mathrm{m})$, a linear molecule can be considered to be a semiflexible polymer. AFM images show the nm-scale structure of DNA adsorbed on the surface. While this technique is useful for imaging, they do not always correspond to molecules in solution, since these images were modulated by the conditions of deposition, they do not always correspond to molecules in solution. FM is an appropriate technique for examining the size of molecules 


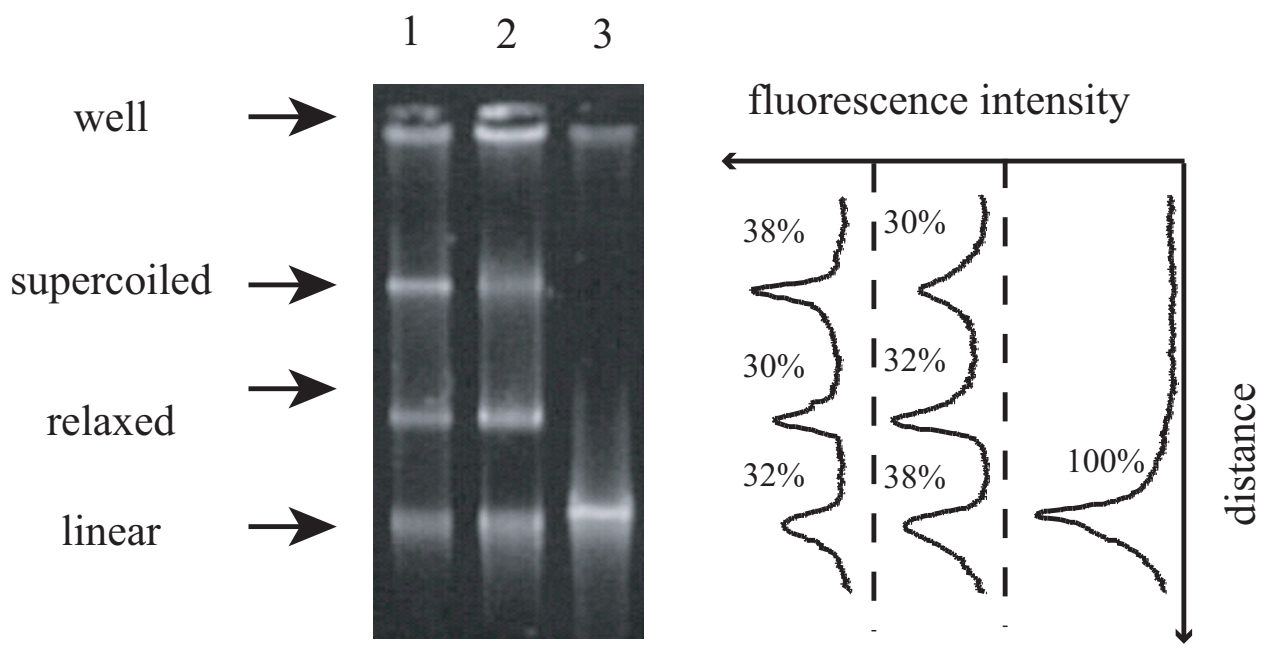

Fig. 1. (left) Electrophoretic characterization of DNA solutions. The 106-kbp DNA forms were resolved by FIGE through an agarose gel $(0.75 \%)$. FIGE was carried out for $22 \mathrm{~h}$ at $283 \mathrm{~K}$ with a field of $3 \mathrm{~V} / \mathrm{cm}$ using $0.5 \times$ TBE buffer. The forward pulse time was $30 \mathrm{~s}$, and the reverse pulse time was $10 \mathrm{~s}$. Lane 1 , sample A; lane 2, sample B; lane 3, sample C. (right) One-dimensional profile of the fluorescence intensity along the lane corresponding to the intensity of the left image and the distribution of forms in each solution.

in solution.

\subsection{FM measurements and analysis of Brownian motion}

We observed the Brownian motion of DNA, as shown in Fig. 3. The fluorescence micrographs of DNA in solution show large conformational fluctuation due to intramolecular Brownian motion of the segments. The linear form tends to be larger than the circular forms. Due to the blurring effect of $0.3-0.5 \mu \mathrm{m}$, the apparent pictures do not give precise information on the actual sizes of individual DNA molecules. In contrast, we can evaluate the hydrodynamic radius of individual molecular chains as a reliable measure. From the Brownian motion trails of the center of mass of DNA, we obtained the time-dependence of the mean-square displacement for each molecule and from these values we obtained the two-dimensional diffusion coefficient $D$ using the following relationship:

$$
<(\mathbf{r}(0)-\mathbf{r}(t))^{2}>=4 D t
$$

where $\mathbf{r}$ is a two-dimensional vector indicating the spatial position of the center of mass of a DNA molecule and the symbol $<>$ denotes a time average in this equation. We used a modified equation with term which is proportional to $t$ squared [11] when a convectional flow was noticed. The hydrodynamic radius $R_{\mathbf{H}}$ is calculated from $D$ based on the Stokes-Einstein relation:

$$
R_{\mathbf{H}}=k_{\mathbf{B}} T / 6 \pi \eta D
$$


(a)

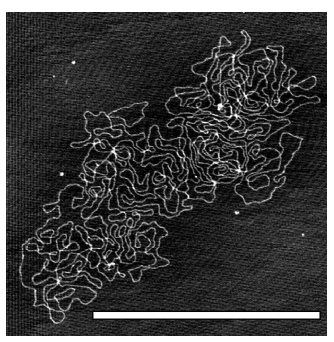

(b)

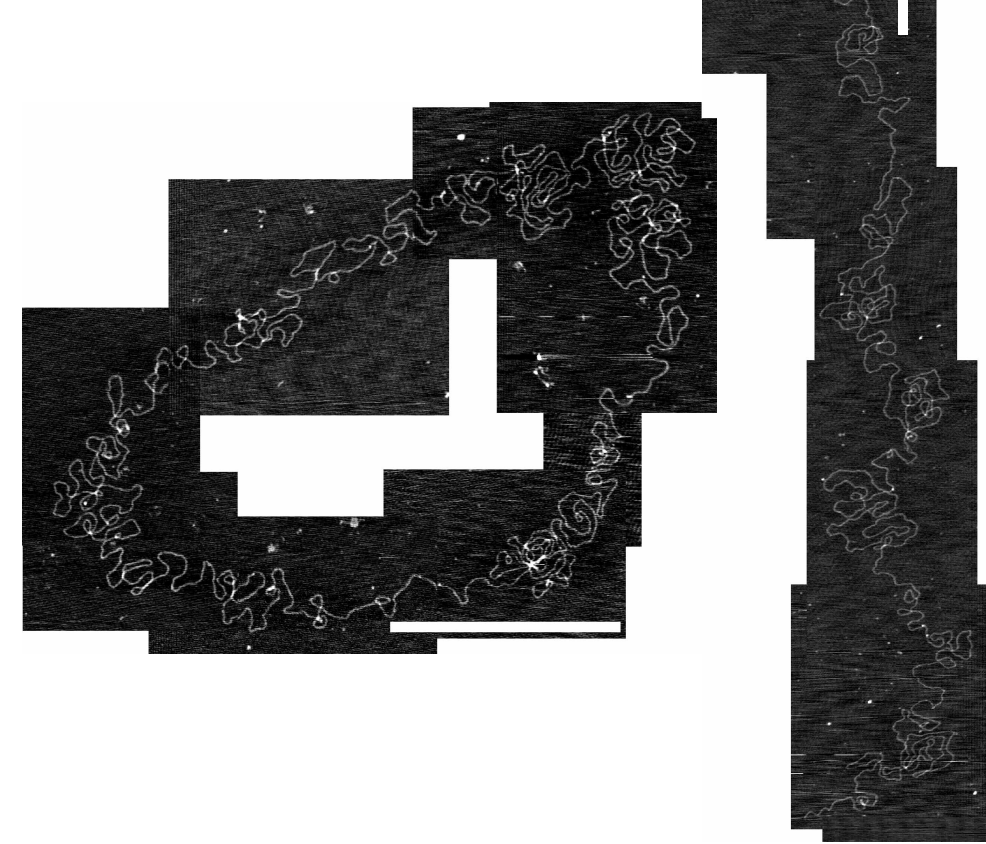

(c)

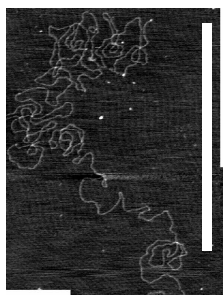

Fig. 2. Typical AFM images of (a) samples A, (b) B and (c) C, where the gyration radii calculated for images were $0.43,1.12$ and $1.57 \mu \mathrm{m}$, respectively. The scale bars represent $1 \mu \mathrm{m}$.

(a)

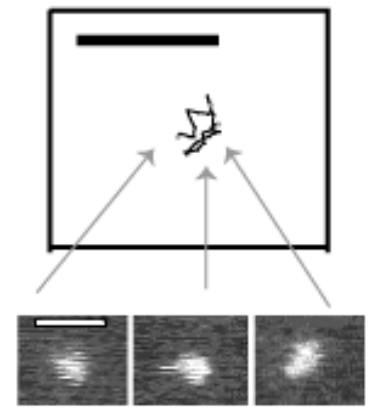

(b)

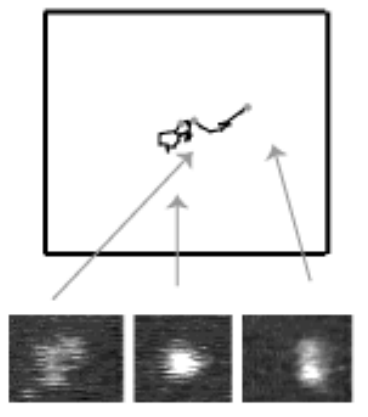

(c)

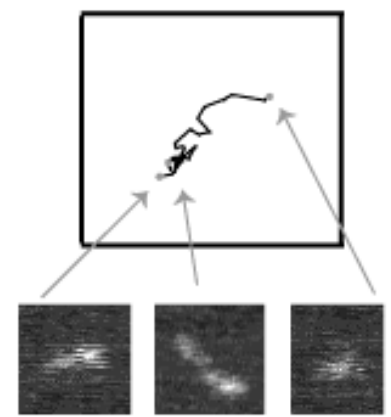

Fig. 3. Analysis of Brownian motion. The Brownian motion trails of the center of mass and typical fluorescence images of individual 106-kbp DNA molecules: (a) sample A, (b) sample B and (c) sample C. The time between the two frames is 0.5 $\mathrm{s}$ and the bar lengths are $2.5 \mu \mathrm{m}$.

where $k_{\mathrm{B}}$ is the Boltzmann constant and $\eta$ is the viscosity of the solvent.

The results of the analysis of Brownian motion are summarized in Fig. 4 as 


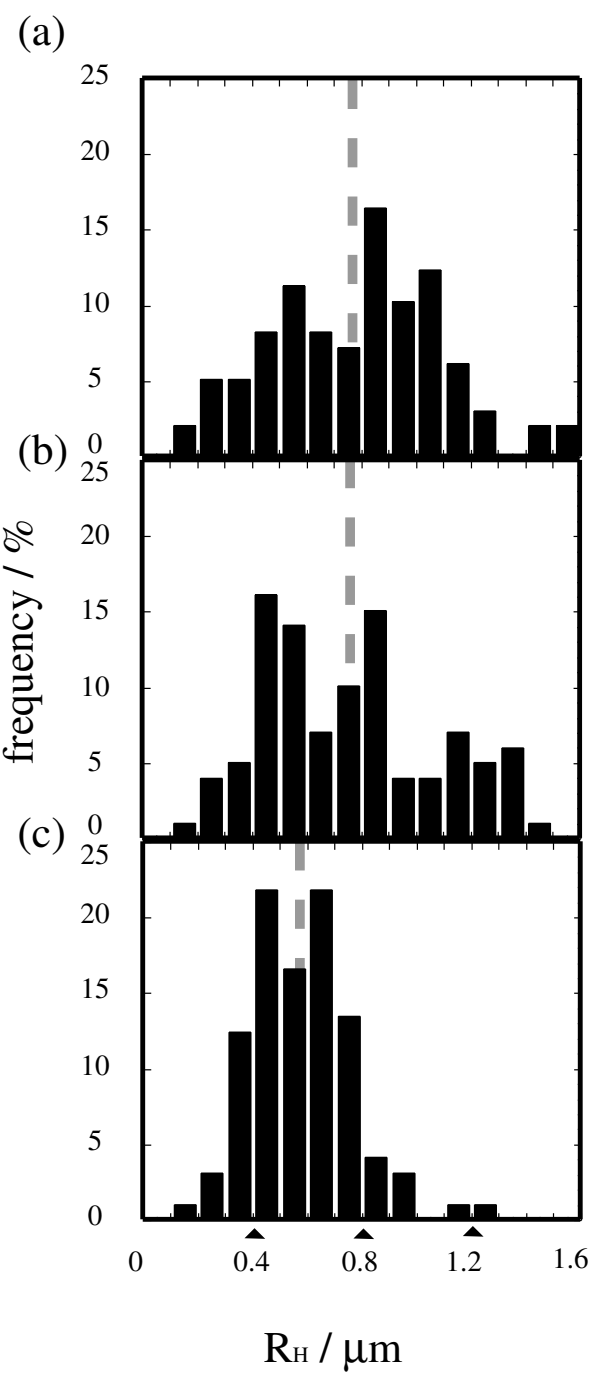

Fig. 4. Histograms of hydrodynamic radii: (a) sample A, (b) sample B, and (c) sample C. $R_{\mathbf{H}}$ (mean average \pm standard deviation) values are $0.78 \pm 0.30,0.76 \pm$ 0.33 and $0.58 \pm 0.19 \mu \mathrm{m}$, respectively.

the histograms of $R_{\mathbf{H}}$ for the three samples; at least 96 DNA molecules of each sample were counted. The mean average hydrodynamic radius measured for sample A $(0.78 \mu \mathrm{m})$ was not much different from that for sample B $(0.76$ $\mu \mathrm{m})$. In contrast, $R_{\mathbf{H}}$ of sample $\mathrm{C}$ was at least $0.2 \mu \mathrm{m}$ smaller than those of samples A and B.

The histogram of $R_{\mathbf{H}}$ for sample $\mathrm{C}$ seems to have a sharp peak compared to those for the other two samples, due to monodispersity of the linear form (Fig. 1). In contrast to sample $\mathrm{C}$, samples $\mathrm{A}$ and $\mathrm{B}$ are the mixtures with three different forms, supercoiled circular, relaxed circular and linear, as indicated in Fig. 1. Due to the fluctuation in the size distribution inherent to the methodology of single DNA observation, it is difficult to reconstruct the distributions of supercoiled circular and relaxed circular DNA in a separate manner. Instead, 
Table 1

Hydrodynamic radius of 106-kbp DNA as deduced from single-chain measurement

\begin{tabular}{cc}
\hline & $<R_{\mathbf{H}}>/ \mu \mathrm{m}$ \\
\hline supercoiled form & 0.94 \\
relaxed form & 0.77 \\
linear form & 0.58 \\
\hline
\end{tabular}

we adopted the procedure to calculate the average value on $R_{\mathbf{H}},<R_{\mathbf{H}}>$, for these two different circular forms. Based on the information on the fractions of individual forms in samples A, B and C, we deduced $\left\langle R_{\mathbf{H}}\right\rangle$ for the different forms through the simple calculation on the $<R_{\mathbf{H}}>$ values. Then, $<R_{\mathbf{H}}>$ of the supercoiled form and that of the relaxed form were determined to be 0.94 and $0.77 \mu \mathrm{m}$, respectively. The $\left\langle R_{\mathbf{H}}>\right.$ values, thus, obtained for each form are summarized in Table. 1. This result is inconsistent with the expectation based on theoretical discussions, as noted in Introduction.

\subsection{Discussion}

The $R_{\mathbf{H}}$ of linear DNA is smaller than that of circular DNA, which is opposite to the above expectation. The fluorescence micrographs and AFM images suggested that the linear form has a larger $R_{\mathrm{g}}$ than the circular forms. However, we consider that additional experiments are needed to confirm these results more precisely. We calculated apparent persistence lengths from the AFM images in Fig. 2. The persistent lengths for (a), (b) and (c) were estimated to be 60, 60 and $80 \mathrm{~nm}$ with an experimental error of $\pm 10 \mathrm{~nm}$; i.e., that for linear DNA is larger than those for circular DNA. This suggests that the degree of swelling in circular DNA is smaller than that in linear DNA, although the difference in the persistence lengths is almost the order of the experimental error. Further studies are awaited to make clear the relative difference in the hydrodynamic radius between the circular and linear chains.

As for the translational Brownian motion of the giant DNA molecule, we have to take account of the possible effect on the anisotropy of the conformation. Although we have tried to evaluate the deviation from isotropic thermal fluctuation on the time-series data, we could not obtain any clear evidence on such anisotropic effect owing to the limited resolution on the FM measurement.

It has been reported that the gyration radius of a circular polymer with excluded volume polymer is larger than that in the absence of topological constraint [15]. It seems to be important to take the topological constraint into account. The dynamics of flexible chains have been understood reasonably well. For a linear excluded-volume polymer, for example, computer simulations 
have been used to study the behavior of $R_{\mathbf{H}}$ [8]. Hydrodynamic interacrion is known to play an essential role for long chains $[10,6]$, which makes the Zimm model superior to the Rouse model in such cases. In the hard sphere system, $R_{\mathbf{H}}$ is larger than $R_{\mathbf{g}}$, whereas $R_{\mathbf{H}}$ is smaller than $R_{\mathbf{g}}$ in a system with low segment density (see Eq.(5)). It may be plausible that the differences in the form and the segment density between linear and circular molecules may affect friction processes. Further theoretical studies are awaited to explain the difference in $R_{\mathbf{H}}$ between linear and circular chains.

\section{Conclusions}

We have found that the hydrodynamic radius $<R_{\mathbf{H}}>$ in linear-form 106-kbp DNA is $\approx 25 \%$ smaller than that in the corresponding circular forms. This result is nontrivial and may be useful for the development of polymer physics in single chains.

\section{Acknowledgements}

This work was supported in part by a Grant-in-aid for the 21st Century COE "Center for Diversity and Universality in Physics" from the Ministry of Education, Culture, Sports, Science and Technology of Japan. T. Nakai is supported by a Research Fellowhip from Japan Society for the Promotion of science for Young scientists $($ No.17 1620$)$. The authors thank the Yukawa Institute for Theoretical Physics at Kyoto University; discussions during the YITP workshop YITP-W-05-04 on "Soft Matter as Structured Materials" were useful to complete this work.

\section{References}

[1] A. Grosberg, A. Khokhlov, Statistical Physics of Macromolecules, American Institute of Physics, New York, NY, 1994.

[2] S. Brown, T. Lenczycki, G. Szamel, Phys. Rev. E 63 (2001) 052801.

[3] A.V. Vologodskii, Mol. Biol. 35 (2001) 240.

[4] L.S. Shlyakhtenko, L.Miloseska, V.N. Potaman, R.R. Sinden, Y. Lyubchenko, Ultramicroscopy 97 (2003) 263.

[5] S.S. Jang, T. Çagin, W.A. Goddard III, J. Chem. Phys. 119 (2003) 1843. 
[6] M. Doi, S. F. Edwards, The Theory of Polymer Dynamics, Oxford, 1986.

[7] B. Dünweg, D. Reith, M. Steinhauser, K. Kremer, J. Chem. Phys. 117 (2002) 914.

[8] A.J.C. Ladd, D. Frenkel, Macromolecules 25 (1992) 3435.

[9] V. Bloomfield, B.H. Zimm, J. Chem. Phys. 44 (1966) 315.

[10] P.G. de Gennes, Scaling Concepts in Polymer Physics, Cornell University Press, Ithaca, NY, 1979.

[11] M. Matsumoto, T. Sakaguchi, H. Kimura, M. Doi, K. Minagawa, Y. Mstsuzawa, K. Yoshikawa, J. Polym. Sci. 30 (1992) 779.

[12] K. Hizume, S. H. Yoshimura, K. Takeyasu, Cell Biochem. Biophys. 40 (2004) 249.

[13] K.D. Cole, C.M. Tallez, Biotechnol. Prog. 18 (2002) 82.

[14] K. Yoshikawa, M. Takahashi, V.V. Vasilevskaya, A.R. Khokhlov, Phys. Rev. Lett. 76 (1996) 3029.

[15] M.K. Shimamura, T. Deguchi, Phys. Rev. E 64 (2001) 020801. 